\title{
A General Inverse Problem for a Memory Kernel in One-Dimensional Viscoelasticity
}

\author{
J. Janno and L. von Wolfersdorf
}

\begin{abstract}
A general inverse problem for the identification of a memory kernel in viscoelasticity in one space dimension is dealt with, where the kernel is represented by a finite sum of products of known spatially dependent functions and unknown time-dependent functions. Using the Laplace transform method an existence and uniqueness theorem for the memory kernel is proved.
\end{abstract}

Keywords: Inverse problems, memory kernels, viscoelasticity, Laplace transform

AMS subject classification: 35R30, 44A10, 45K05, 73F15

\section{Introduction}

Time-dependent memory kernels in viscoelasticity can be identified by solving related inverse problems as had been done, for instance, in the papers [4 - 5] by Grasselli and in our papers [7 - 9] (see also [12]).

In our recent paper [10] such an inverse problem has been treated for a viscoelastic material in one space dimension with memory kernel which can be represented by a finite sum of $N$ products of known space-dependent functions with unknown time-dependent functions. These kernels are looked on as mathematical approximations of a general kernel depending on both the space and the time variable. The known space-dependent functions model the spatial inhomogenities of the medium whereas the time structure of the kernel is not prescribed a priori. The unknown time-dependent functions are recovered by additional measurements of the stress in fixed points. A

J. Janno: Inst. Cybern. at Tallinn Techn. Univ., 12618 Tallinn, Estonia; janno@ioc.ee. The paper was written during a visit of the author at the Freiberg Univ. of Mining and Technology with a grant of DAAD (Germany) from July to August 2001, also supported by the Estonian Science Foundation (Grant No. 4345).

L. von Wolfersdorf: Techn. Univ. Bergakademie Freiberg, Fak. Math. \& Inf., D09596 Freiberg; wolfersd@mathe.tu-freiberg.de

ISSN 0232-2064 / \$2.50 C Heldermann Verlag Berlin 
corresponding problem in higher space dimension has been discussed recently in the paper [6] by Janno and Lorenzi.

In this paper a general inverse problem for such memory kernels in one space dimension with observations of the displacement in inner points for different initial and boundary values and different source terms is treated. This corresponds to additional measurements for the displacement obtained from several experiments with the same material as usually occurs in engineering practice.

As in [10] we use the Laplace transform method which requires now some additional techniques and stronger assumptions on the data because of the higher degree of ill-posedness of the problem. The existence and uniqueness of the memory kernel is then proved by a contraction argument again. In contrast to [10] we give here full proofs of the corresponding theorem and the related lemma.

We remark that identification problems in several space dimensions for other classes of memory kernels in diffusion and viscoelasticity depending on time and some local coordinates are dealt with in the papers $[1-2,11]$ by Lorenzi and co-authors.

In Section 2 we formulate the direct and inverse viscoelasticity problem and in Section 3 we transform these problems by the Laplace transform. After preparations in Sections 4 and 5 on the properties of the Green function in the Laplace transform domain and on an estimation of the Laplace transform of the solution of the direct problem, respectively, the existence and uniqueness theorem for the solution to the inverse problem is derived in Section 6 .

\section{Statement of problem}

In the linear theory of wave propagation in a non-homogeneous viscoelastic bar the Boltzmann stress-strain relation

$$
\sigma(x, t)=\beta(x) \epsilon(x, t)-\int_{-\infty}^{t} m(x, t-\tau) \epsilon(x, \tau) d \tau
$$

in $0<x<1$ and $t>0$ between the strain $\epsilon$ and the stress $\sigma$ is supposed as a constitutive relation, where $m$ is the relaxation memory kernel. Further, there hold the relation $\epsilon=u_{x}$ with the displacement $u$ and the momentum equation

$$
\rho(x) u_{t t}=\sigma_{x}+r(x, t) \quad(x \in(0,1), t>0)
$$

with the mass density $\rho$ and the outer force density $r$. The given positive functions $\beta$ and $\rho$ are supposed as sufficiently smooth in $[0,1]$. The force 
density $r$ is taken as a bounded continuous function. Further, we assume $u(x, t) \equiv 0$ for $t<0$.

In the following we consider the viscoelastic system characterized by $\beta, m$ and $\rho$ in $(2.1)-(2.2)$ for force terms $r=r_{j} \quad(j=1, \ldots, l)$. Then for the corresponding displacements $u=u_{j} \quad(j=1, \ldots, l)$ the generalized wave equations

$$
\rho(x) u_{j, t t}=\left(\beta(x) u_{j, x}\right)_{x}-\left(\int_{0}^{t} m(x, t-\tau) u_{j, x}(x, \tau) d \tau\right)_{x}+r_{j}(x, t)
$$

in $(0,1) \times(0, \infty)$ hold. Moreover, the functions $u_{j}$ satisfy the initial and boundary conditions

$$
\left.\begin{array}{rl}
u_{j}(x, 0) & =\varphi_{j}(x) \\
u_{j, t}(x, 0) & =\psi_{j}(x)
\end{array}\right\} \quad(x \in(0,1))
$$

and

$$
\left.\begin{array}{l}
u_{j}(0, t)=f_{j}(t) \\
u_{j}(1, t)=g_{j}(t)
\end{array}\right\} \quad(t>0)
$$

with given smooth functions $\varphi_{j}, \psi_{j}(j=1, \ldots, l)$ on $[0,1]$ and bounded continuous functions $f_{j}, g_{j}(j=1, \ldots, l)$ on $[0, \infty)$. Without loss of generality we have taken the same starting point $t=0$ for $j=1, \ldots, l$.

In what follows, we take the kernel $m$ in the form

$$
m(x, t)=\sum_{k=1}^{N} \mu_{k}(x) m_{k}(t)
$$

where $\mu_{k}$ are given continuous functions on $[0,1]$ and $m_{k}$ are unknown memory kernels depending on $t \in(0, \infty)$.

Our aim is to determine the unknown $m_{k}$ by additional measurements of the displacements $u_{j}$ in inner points $x_{i j}\left(i=1, \ldots, N_{j}\right)$ where $\sum_{j=1}^{l} N_{j}=$ $N \quad\left(N_{j} \geq 1\right)$. So, in the inverse problem we have the additional conditions

$$
u_{j}\left(x_{i j}, t\right)=h_{j i}(t) \quad\left(i=1, \ldots, N_{j} ; j=1, \ldots, l\right)
$$

where $0<x_{i j}<1$ are the observation points and $h_{j i}$ are given ounded continuous functions on $[0, \infty)$. 


\section{Application of the Laplace transformation}

As in [10] (see also [9]) we apply the Laplace transformation to equations (2.3) with (2.6) and initial conditions (2.4). Then, for the Laplace transform of $u_{j} \quad(j=1, \ldots, l)$,

$$
U_{j}(x, p)=\mathcal{L}_{t \rightarrow p} u_{j}=\int_{0}^{\infty} e^{-p t} u_{j}(x, t) d t \quad(\operatorname{Re} p>\eta)
$$

with some real number $\eta$, the equation

$$
\begin{aligned}
\rho(x) & {\left[p^{2} U_{j}(x, p)-p \varphi_{j}(x)-\psi_{j}(x)\right] } \\
& =\left(\beta(x) U_{j, x}(x, p)\right)_{x}-\sum_{k=1}^{N} M_{k}(p)\left(\mu_{k}(x) U_{j, x}(x, p)\right)_{x}+R_{j}(x, p)
\end{aligned}
$$

holds for $x \in(0,1)$, where $M_{k}=\mathcal{L}_{t \rightarrow p} m_{k}$ and $R_{j}=\mathcal{L}_{t \rightarrow p} r_{j}$ (cf. [3]).

The boundary conditions (2.5) are transformed to

$$
\left.\begin{array}{l}
U_{j}(0, p)=F_{j}(p) \\
U_{j}(1, p)=G_{j}(p)
\end{array}\right\} \quad(\operatorname{Re} p>\eta)
$$

where $F_{j}=\mathcal{L}_{t \rightarrow p} f_{j}$ and $G_{j}=\mathcal{L}_{t \rightarrow p} g_{j} \quad(j=1, \ldots, l)$. Further, the additional conditions (2.7) transform to

$$
U_{j}\left(x_{i j}, p\right)=H_{j i}(p) \quad\left(\operatorname{Re} p>\eta ; i=1, \ldots, N_{j}, j=1, \ldots, l\right)
$$

where $H_{j i}=\mathcal{L}_{t \rightarrow p} h_{j i}$.

Writing equations (3.1) in the form

$$
\begin{aligned}
\left(L U_{j}\right)(x, p)= & \sum_{k=1}^{N} M_{k}(p)\left(\mu_{k}(x) U_{j, x}(x, p)\right)_{x}-\rho(x)\left[p \varphi_{j}(x)+\psi_{j}(x)\right] \\
& -R_{j}(x, p)
\end{aligned}
$$

for $j=1, \ldots, l$ with the differential operator

$$
(L U)(x, p)=\left(\beta(x) U_{x}(x, p)\right)_{x}-\rho(x) p^{2} U(x, p) \quad(0<x<1),
$$

the solution $U_{j}$ of equation (3.4) with (3.5) can be represented by

$$
U_{j}(x, p)=\sum_{k=1}^{N} M_{k}(p) \int_{0}^{1} G(x, y ; p)\left(\mu_{k}(y) U_{j, y}(y, p)\right)_{y} d y-\chi_{j}(x, p)
$$


where $G(x, y ; p)$ denotes the Green function of $L$ with boundary conditions of the first kind and

$$
\begin{aligned}
\chi_{j}(x, p)= & \int_{0}^{1} G(x, y ; p)\left[\rho(y)\left(p \varphi_{j}(y)+\psi_{j}(y)\right)+R_{j}(y, p)\right] d y \\
& +G_{y}(x, 0 ; p) \beta(0) F_{j}(p)-G_{y}(x, 1 ; p) \beta(1) G_{j}(p)
\end{aligned}
$$

for $0 \leq x \leq 1$.

We differentiate (3.6) with respect to $x$ and integrate by parts taking the well-known jump relation for $G_{x}$

$$
G_{x}(x, x-0 ; p)-G_{x}(x, x+0 ; p)=\frac{1}{\beta(x)} \quad(0 \leq x \leq 1)
$$

into account. Then we obtain the integral equation for $U_{j, x}(j=1, \ldots, l)$

$$
\begin{aligned}
U_{j, x}(x, p)= & \frac{1}{\beta(x)} \sum_{k=1}^{N} M_{k}(p) \mu_{k}(x) U_{j, x}(x, p) \\
& -\sum_{k=1}^{N} M_{k}(p) \int_{0}^{1} G_{x y}(x, y ; p) \mu_{k}(y) U_{j, y}(y, p) d y-\chi_{j, x}(x, p)
\end{aligned}
$$

in $0 \leq x \leq 1$. The direct problem $(2.3)-(2.5)$ is reduced to integral equation (3.9) for $U_{j, x}(j=1, \ldots, l)$. From $U_{j, x}$ one can get $U_{j}$ itself by integration in view of one of the boundary relations (3.2) or by means of formula (3.6). In dealing with the inverse problem we choose the second way in the following.

By (3.6) the additional conditions of the inverse problem take the form of the integral conditions

$$
\sum_{k=1}^{N} M_{k}(p) \int_{0}^{1} G\left(x_{i j}, y ; p\right)\left(\mu_{k}(y) U_{j, y}(y, p)\right)_{y} d y=H_{j i}(p)+\chi_{j}\left(x_{i j}, p\right)
$$

for $i=1, \ldots, N_{j}$ and $j=1, \ldots, l$. We multiply these equations for $M_{k}(p) \quad(k=$ $1, \ldots, N)$ by $p^{3}$ and write them as

$$
\begin{aligned}
& \sum_{k=1}^{N} M_{k}(p) \int_{0}^{1} p^{2} G\left(x_{i j}, y ; p\right)\left[p\left(\mu_{k}(y) U_{j, y}(y, p)\right)_{y}-\left(\mu_{k}(y) \varphi_{j}^{\prime}(y)\right)^{\prime}\right] d y \\
& +\sum_{k=1}^{N} M_{k}(p) \int_{0}^{1} p^{2} G\left(x_{i j}, y ; p\right)\left(\mu_{k}(y) \varphi_{j}^{\prime}(y)\right)^{\prime} d y=p^{3}\left[H_{j i}(p)+\chi_{j}\left(x_{i j}, p\right)\right] .
\end{aligned}
$$


We define the coefficients

$$
\gamma_{i j ; k}=\frac{1}{\rho\left(x_{i j}\right)}\left[\left(\mu_{k}(x) \varphi_{j}^{\prime}(x)\right)^{\prime}\right]_{x=x_{i j}}
$$

$\left(i=1, \ldots, N_{j}, j=1, \ldots, l, k=1, \ldots, N\right)$ and integrate by parts the first integral in (3.11). This yields the system of $N$ equations for the functions $M_{k}(p) \quad(k=$ $1, \ldots, N)$

$$
\begin{aligned}
& \sum_{k=1}^{N} \gamma_{i j ; k} M_{k}(p)-\sum_{k=1}^{N} M_{k}(p)\left[\int_{0}^{1} p^{2} G\left(x_{i j}, y ; p\right)\left(\mu_{k}(y) \varphi_{j}^{\prime}(y)\right)^{\prime} d y+\gamma_{i j ; k}\right] \\
& +\sum_{k=1}^{N} M_{k}(p) \int_{0}^{1} p^{2} G_{y}\left(x_{i j}, y ; p\right) \mu_{k}(y)\left[p U_{j, y}(y, p)-\varphi_{j}^{\prime}(y)\right] d y=\Psi_{j i}(p)
\end{aligned}
$$

where $i=1, \ldots, N_{j}, j=1, \ldots, l$ and

$$
\Psi_{j i}(p)=-p^{3}\left[H_{j i}(p)+\chi_{j}\left(x_{i j}, p\right)\right] .
$$

In equations (3.13) the first sum is the main part of the left-hand side for $\operatorname{Re} p \rightarrow+\infty$ since

$$
\int_{0}^{1} p^{2} G\left(x_{i j}, y ; p\right)\left(\mu_{k}(y) \varphi_{j}^{\prime}(y)\right)^{\prime} d y \rightarrow-\gamma_{i j ; k}
$$

and

$$
p U_{j, x}(x, p) \rightarrow \varphi_{j}^{\prime}(x) \quad(0<x<1)
$$

for $\operatorname{Re} p \rightarrow+\infty$.

Asymptotic relation (3.15) is a consequence of the second part of Lemma 2 below. Limit (3.16) follows from the first initial condition (2.4) by a wellknown relation from the Laplace transform theory where the functions $\varphi_{j}(j=$ $1, \ldots, l)$ are assumed as being continuously differentiable (cf. [3: Theorem $24.5])$.

Hence inverse problem (2.3) - (2.7) is reduced to the system of equations (3.13) for the functions $M_{k} \quad(k=1, \ldots, N)$ where $U_{j, x}=U_{j, x}[M] \quad(M=$ $\left.\left(M_{1}, \ldots M_{N}\right), j=1, \ldots, l\right)$ are the solutions of integral equations (3.9).

In the following sometimes we also use the new running index $n$ defined by

$$
n=\sum_{\alpha=1}^{j-1} N_{\alpha}+i \quad\left(j \in\{1, \ldots, l\}, i=1, \ldots, N_{j}\right)
$$

and going from 1 to $N$ instead of the two indices $i, j$. In this case we put $\gamma_{n k}=\gamma_{i j ; k}$. We assume the regularity condition

$$
\operatorname{det} \Gamma \neq 0
$$

for the coefficient matrix $\Gamma=\left(\left(\gamma_{n k}\right)\right)_{n, k=1}^{N}$. 


\section{Properties of the Green function}

The Green function $G$ is defined by the expression (cf. [10])

$$
G(x, y ; p)= \begin{cases}\frac{1}{\Delta_{0} \beta(0)} \psi_{2}(y) \psi_{1}(x) & \text { for } 0 \leq x \leq y \leq 1 \\ \frac{1}{\Delta_{0} \beta(0)} \psi_{1}(y) \psi_{2}(x) & \text { for } 0 \leq y \leq x \leq 1\end{cases}
$$

where $\psi_{\nu}(x)=\psi_{\nu}(x, p) \quad(\nu=1,2)$ are the solutions of $L \psi=0$ which satisfy the initial conditions

$$
\left.\left.\begin{array}{l}
\psi_{1}(0)=0 \\
\psi_{1}^{\prime}(0)=\frac{b(0)}{X}
\end{array}\right\} \quad \text { and } \quad \begin{array}{l}
\psi_{2}(1)=0 \\
\psi_{2}^{\prime}(1)=\frac{b(1)}{X}
\end{array}\right\}
$$

where

$$
X=\int_{0}^{1} \sqrt{\frac{\rho}{\beta}} d x \quad \text { and } \quad b(x)=\rho(x)^{\frac{1}{4}} \beta(x)^{-\frac{3}{4}}
$$

and the Wronski determinant at zero

$$
\Delta_{0}=\Delta_{0}(p)=-\psi_{1}^{\prime}(0) \psi_{2}(0) .
$$

The Green function $G$ has the asymptotic representation (cf. [10] again)

$$
G(x, y ; p)=\frac{1}{C_{0}} \frac{1}{a(x) a(y)} \frac{1}{s^{2}} \begin{cases}\sinh s z \cdot \sinh s(w-1)+O_{1} & \text { for } x \leq y \\ \sinh s w \cdot \sinh s(z-1)+O_{2} & \text { for } y \leq x\end{cases}
$$

where $s=X p$,

$$
\begin{aligned}
C_{0} & =\frac{1}{X s} \sinh s+O\left(\frac{e^{s}}{s^{2}}\right) \quad(\operatorname{Re} p \rightarrow+\infty) \\
a(x) & =(\rho(x) \beta(x))^{\frac{1}{4}} \\
z & =\frac{1}{X} \int_{0}^{x} \sqrt{\frac{\rho(\xi)}{\beta(\xi)}} d \xi, \quad w=\frac{1}{X} \int_{0}^{y} \sqrt{\frac{\rho(\eta)}{\beta(\eta)}} d \eta
\end{aligned}
$$

and the $O$-terms

$$
\begin{aligned}
& O_{1}=O\left(\frac{e^{s(1-w+z)}}{s}\right) \\
& O_{2}=O\left(\frac{e^{s(w+1-z)}}{s}\right)
\end{aligned} \quad(\operatorname{Re} p \rightarrow+\infty)
$$

holding uniformly in $x$ and $y$. For the derivatives $G_{x}$ and $G_{y}$ we have the representations

$$
\begin{aligned}
& G_{x}(x, y ; p)=\frac{1}{C_{0} X} \frac{b(x)}{a(y)} \frac{1}{s} \begin{cases}\cosh s z \cdot \sinh s(w-1)+O_{3} & \text { for } x<y \\
\sinh s w \cdot \cosh s(1-z)+O_{4} & \text { for } y<x\end{cases} \\
& G_{y}(x, y ; p)=\frac{1}{C_{0} X} \frac{b(y)}{a(x)} \frac{1}{s} \begin{cases}\sinh s z \cdot \cosh s(w-1)+O_{5} & \text { for } x<y \\
\cosh s w \cdot \sinh s(z-1)+O_{6} & \text { for } y<x\end{cases}
\end{aligned}
$$

where the terms $O_{3}, O_{5}$ behave like $O_{1}$ and the terms $O_{4}, O_{6}$ like $O_{2}$, respectively, for $\operatorname{Re} p \rightarrow+\infty$.

At first we have 
Lemma 1. If $\beta, \rho \in C^{2}[0,1]$ and $\beta, \rho>0$ in $[0,1]$, then the estimations

$$
\begin{aligned}
& C_{1}:=\sup _{\substack{0 \leq x \leq 1 \\
\operatorname{Re} p>0}}\left(\operatorname{Re} p \int_{0}^{1}\left|G_{y}(x, y ; p)\right| d y\right)<+\infty \\
& C_{2}:=\sup _{\substack{0 \leq x \leq 1 \\
\operatorname{Re} p>0}}\left(\frac{\operatorname{Re} p}{|p|} \int_{0}^{1}\left|G_{x y}(x, y ; p)\right| d y\right)<+\infty
\end{aligned}
$$

hold.

Proof. The second estimation (4.10) has already been proved in [10]. In analogous way we prove the first estimation (4.9). Observing the relations

$$
\left|e^{s z}\right|=e^{\operatorname{Re} s \cdot z}, \quad|\cosh s z| \leq \cosh (\operatorname{Re} s \cdot z), \quad|\sinh s| \geq \sinh (\operatorname{Re} s)
$$

from (4.8) we obtain

$$
\begin{aligned}
\int_{0}^{1}\left|G_{y}(x, y ; p)\right| d y \leq & C \frac{1}{\operatorname{Re} s} \frac{1}{\sinh (\operatorname{Re} s)}\{\cosh (\operatorname{Re} s \cdot z) \sinh (\operatorname{Re} s \cdot[1-z]) \\
& +\sinh (\operatorname{Re} s \cdot z) \cosh (\operatorname{Re} s \cdot[1-z])\} \\
= & C \frac{1}{\operatorname{Re} p}
\end{aligned}
$$

with some constant $C>0$. This implies (4.9)

Further, the following lemma is valid.

Lemma 2. Let be $\beta, \rho \in C^{2}[0,1]$ and $\beta, \rho>0$ in $[0,1]$. Then the following assertions hold:

(i) For any $v \in C^{3}[0,1]$ such that $v^{\prime}(0)=v^{\prime}(1)=0$ the estimate

$$
\sup _{\substack{0 \leq x \leq 1 \\ \operatorname{Re} p>0}}\left|p^{2}\left(\int_{0}^{1} G_{x y}(x, y ; p) v(y) d y-\frac{v(x)}{\beta(x)}\right)\right| \leq C_{3}\left\|v^{\prime \prime}\right\|_{C^{1}[0,1]}
$$

is valid, where $C_{3}>0$ is a constant.

(ii) For any $v \in C^{2}[0,1]$ such that $v(0)=v(1)=0$ the estimate

$$
\sup _{\substack{0 \leq x \leq 1 \\ \operatorname{Re} p>0}}\left|p\left(\int_{0}^{1} p^{2} G(x, y ; p) v(y) d y+\frac{v(x)}{\rho(x)}\right)\right| \leq C_{4}\left\|v^{\prime}\right\|_{C^{1}[0,1]}
$$

is valid where $C_{4}>0$ is a constant.

Here the norm in $C^{1}$ is defined by

$$
\|w\|_{C^{1}[0,1]}=\max _{0 \leq x \leq 1}|w(x)|+\max _{0 \leq x \leq 1}\left|w^{\prime}(x)\right| .
$$

The first part (i) of Lemma 2 is analogous to the corresponding one in $[10$ : Lemma 1] where the right-hand side could be given in the norm $\left\|v^{\prime}\right\|_{C^{1}[0,1]}$, too. 
Proof of Lemma 2. First we prove assertion (i). For this aim we split the integral $\int_{0}^{1} G_{x y}(x, y ; p) v(y) d y$ into the integrals from 0 to $x$ and from $x$ to 1 . Integrating both integrals by parts, in view of the jump relation for $G_{x}$ (3.8) and the equalities $G_{x}(x, 0 ; p)=G_{x}(x, 1 ; p)=0 \quad(0<x<1)$ following from the boundary conditions $G(x, 0 ; p)=G(x, 1 ; p)=0 \quad(0 \leq x \leq 1)$ for $G$ itself, we at first obtain the relation

$$
\int_{0}^{1} G_{x y}(x, y ; p) v(y) d y-\frac{v(x)}{\beta(x)}=-\int_{0}^{1} G_{x}(x, y ; p) v^{\prime}(y) d y .
$$

Before integrating by parts a second and a third time, we introduce the functions

$$
K(x, y ; p)=\int_{x}^{y} G_{x}(x, \eta ; p) d \eta+\frac{b(x) \bar{b}(x)}{C_{0} s^{2}} \cosh s z \cdot \cosh s(z-1)
$$

where

$$
\bar{b}(x)=\sqrt{\frac{\beta(x)}{\rho(x)}} \frac{1}{a(x)}=\rho(x)^{-\frac{3}{4}} \beta(x)^{\frac{1}{4}}
$$

and

$$
\bar{K}(x, y ; p)= \begin{cases}\int_{1}^{y} K(x, \eta ; p) d \eta & \text { for } x<y \\ \int_{0}^{y} K(x, \eta ; p) d \eta & \text { for } y<x\end{cases}
$$

with $\bar{K}(x, 0 ; p)=\bar{K}(x, 1 ; p)=0 \quad(0<x<1)$. Then, in view of $v^{\prime}(0)=$ $v^{\prime}(1)=0$, from (4.14) we further have

$$
\begin{aligned}
& \int_{0}^{1} G_{x y}(x, y ; p) v(y) d y-\frac{v(x)}{\beta(x)} \\
& \quad=\int_{0}^{1} K(x, y ; p) v^{\prime \prime}(y) d y \\
& \quad=[\bar{K}(x, x-0 ; p)-\bar{K}(x, x+0 ; p)] v^{\prime \prime}(x)-\int_{0}^{1} \bar{K}(x, y ; p) v^{\prime \prime \prime}(y) d y .
\end{aligned}
$$

In obtaining the asymptotic relation for $\bar{K}$ we first derive the one for $K$. For $x<y$, by (4.7) from (4.15) we have

$$
\begin{aligned}
K(x, y ; p)= & \frac{1}{C_{0} X} \frac{b(x)}{s}\left[\cosh s z \int_{x}^{y} \frac{1}{a(\eta)} \sinh s(\tilde{w}-1) d \eta\right. \\
& \left.+\int_{x}^{y} O_{3}\left(\frac{e^{s(1-\tilde{w}+z)}}{s}\right) \frac{1}{a(\eta)} d \eta\right]+\frac{b(x) \bar{b}(x)}{C_{0} s^{2}} \cosh s z \cdot \cosh s(z-1)
\end{aligned}
$$


where $\tilde{w}=\frac{1}{X} \int_{0}^{\eta} \sqrt{\frac{\rho(\tau)}{\beta(\tau)}} d \tau$ and $d \tilde{w}=\frac{1}{X} \int_{0}^{\eta} \sqrt{\frac{\rho(\eta)}{\beta(\eta)}} d \eta$ and hence

$$
\begin{aligned}
K(x, y ; p)= & \frac{b(x)}{C_{0} s}\left[\cosh s z\left(\int_{z}^{w} \bar{b}(\eta) \sinh s(\tilde{w}-1) d \tilde{w}+\frac{\bar{b}(x)}{s} \cosh s(z-1)\right)\right. \\
& \left.+\int_{z}^{w} \bar{b}(\eta) O_{3}\left(\frac{e^{s(1-\tilde{w}+z)}}{s^{2}}\right) d \tilde{w}\right] .
\end{aligned}
$$

Integrating by parts, we then obtain

$$
K(x, y ; p)=\frac{b(x) \bar{b}(y)}{C_{0} s^{2}}\left[\cosh s z \cdot \cosh s(w-1)+O\left(\frac{e^{s(1-w+z)}}{s}\right)\right] .
$$

For $y<x$ in an analogous way we get a corresponding expression, so that finally we obtain

$$
K(x, y ; p)=\frac{b(x) \bar{b}(y)}{C_{0} s^{2}} \begin{cases}\cosh s z \cdot \cosh s(w-1)+O_{7} & \text { for } x \leq y \\ \cosh s w \cdot \cosh s(1-z)+O_{8} & \text { for } y \leq x\end{cases}
$$

where the term $O_{7}$ behaves like $O_{1}$ and the term $O_{8}$ like $O_{2}$ again. In a similar way from (4.17) and (4.19) we derive the asymptotic representation for $\bar{K}$

$$
\bar{K}(x, y ; p)=\frac{X b(x) \hat{b}(y)}{C_{0} s^{3}} \begin{cases}\cosh s z \cdot \sinh s(w-1)+O_{9} & \text { for } x<y \\ \sinh s w \cdot \cosh s(1-z)+O_{10} & \text { for } y<x\end{cases}
$$

where

$$
\hat{b}(x)=\sqrt{\frac{\beta(x)}{\rho(x)}} \bar{b}(x)=\rho(x)^{-\frac{5}{4}} \beta(x)^{\frac{3}{4}}
$$

and $O_{9}$ and $O_{10}$ behave like $O_{1}$ and $O_{2}$, respectively.

In view of (4.5) relation (4.20) yields the estimate

$$
|\bar{K}(x, y ; p)| \leq \frac{\bar{C}}{|p|^{2}} \quad(0 \leq x, y \leq 1)
$$

with some constant $\bar{C}>0$. Hence representation (4.18) implies assertion (4.12) in (i).

Next let us prove assertion (ii). To this end we define the related functions

$$
H(x, y ; p)= \begin{cases}\int_{1}^{y} G(x, \eta ; p) d \eta+\frac{X}{C_{0} a(x)} \frac{\bar{b}(1)}{s^{3}} \sinh s z & \text { for } x<y \\ \int_{0}^{y} G(x, \eta ; p) d \eta+\frac{X}{C_{0} a(x)} \frac{\bar{b}(0)}{s^{3}} \sinh s(z-1) & \text { for } y<x\end{cases}
$$

and

$$
\bar{H}(x, y ; p)= \begin{cases}\int_{1}^{y} H(x, \eta ; p) d \eta & \text { for } x<y \\ \int_{0}^{y} H(x, \eta ; p) d \eta & \text { for } y<x .\end{cases}
$$


In view of $v(0)=v(1)=0$ we have

$$
\begin{aligned}
\int_{0}^{1} G(x, y ; p) v(y) d y= & \Delta(x ; p) v(x)-\int_{0}^{1} H(x, y ; p) v^{\prime}(y) d y \\
= & \Delta(x ; p) v(x)-[\bar{H}(x, x-0 ; p)-\bar{H}(x, x+0 ; p)] v^{\prime}(x) \\
& +\int_{0}^{1} \bar{H}(x, y ; p) v^{\prime \prime}(y) d y
\end{aligned}
$$

where

$$
\Delta(x ; p)=H(x, x-0 ; p)-H(x, x+0 ; p) .
$$

In a similar way as above we derive the asymptotic relations

$$
\begin{aligned}
& H(x, y ; p)=\frac{X \bar{b}(y)}{C_{0} a(x)} \frac{1}{s^{3}} \begin{cases}\sinh s z \cdot \cosh s(w-1)+O_{11} & \text { for } x<y \\
\cosh s w \cdot \sinh s(z-1)+O_{12} & \text { for } y<x\end{cases} \\
& \bar{H}(x, y ; p)=\frac{X^{2} \hat{b}(y)}{C_{0} a(x)} \frac{1}{s^{4}} \begin{cases}\sinh s z \cdot \sinh s(w-1)+O_{13} & \text { for } x \leq y \\
\sinh s w \cdot \sinh s(z-1)+O_{14} & \text { for } y \leq x\end{cases}
\end{aligned}
$$

where the $O$-terms $O_{11}, O_{13}$ behave like $O_{1}$ and $O_{12}, O_{14}$ like $O_{2}$, respectively. From (4.27) for the function $\Delta$ defined by (4.26) we obtain

$\Delta=\frac{X \bar{b}(x)}{C_{0} a(x)} \frac{1}{s^{3}}\left[-\sinh s+O\left(\frac{e^{s}}{s}\right)\right]=\frac{1}{p^{2}}\left[-\frac{1}{\rho(x)}+O\left(p^{-1}\right)\right]$ uniformly in $x$

taking into account relations (4.5) - (4.6), (4.16) and $s=X p$. Observing (4.5) again, from (4.28) we get the estimate

$$
|\bar{H}(x, y ; p)| \leq \frac{\widehat{C}}{|p|^{3}} \quad(0 \leq x, y \leq 1)
$$

with some constant $\widehat{C}>0$. Finally, using (4.29) and (4.30), representation (4.25) yields us assertion (4.13) in (ii) with the norm of $v$ in $C^{2}[0,1]$ or, equivalently, with the norm of $v^{\prime}$ in $C^{1}[0,1]$ due to condition $v(0)=v(1)=0$. Lemma 2 is completely proved 


\section{Estimation of the solution to the direct problem}

In equations (3.13) the functions

$$
B_{j}=B_{j}[M]=p U_{j, x}(x, p)-\varphi_{j}^{\prime}(x) \quad\left(M=\left(M_{1}, \ldots, M_{N}\right)\right)
$$

occur where $U_{j}(j=1, \ldots, l)$ are the solutions of the direct problem. By (3.9) the functions $B_{j}$ satisfy the integral equations

$$
\begin{aligned}
B_{j}(x, p)= & \frac{1}{\beta(x)} \sum_{k=1}^{N} M_{k}(p) \mu_{k}(x) \cdot B_{j}(x, p) \\
& -\sum_{k=1}^{N} M_{k}(p) \int_{0}^{1} G_{x y}(x, y ; p) \mu_{k}(y) B_{j}(y, p) d y \\
& +\sum_{k=1}^{N} M_{k}(p) \Phi_{j k}(x, p)-\Lambda_{j}(x, p)
\end{aligned}
$$

where

$$
\begin{aligned}
\Phi_{j k}(x, p) & =\frac{\mu_{k}(x)}{\beta(x)} \varphi_{j}^{\prime}(x)-\int_{0}^{1} G_{x y}(x, y ; p) \mu_{k}(y) \varphi_{j}^{\prime}(y) d y \\
\Lambda_{j}(x, p) & =p \chi_{j, x}(x, p)+\varphi_{j}^{\prime}(x)
\end{aligned}
$$

with $\chi_{j}(j=1, \ldots, l)$ given by $(3.7)$.

The following spaces for complex-valued scalar and vector functions are used as in [10]:

$$
\mathcal{A}_{\gamma, \sigma}=\left\{V: V(p) \text { holomorphic on } \operatorname{Re} p>\sigma \text { and }\|V\|_{\gamma, \sigma}<\infty\right\} \quad(\gamma, \sigma>0)
$$

with norm $\|V\|_{\gamma, \sigma}=\sup _{\operatorname{Re} p>\sigma}|p|^{\gamma}|V(p)|$ and

$$
\left(\mathcal{A}_{\gamma, \sigma}\right)^{N}=\left\{V=\left(V_{1}, \ldots, V_{N}\right): V_{k}(p) \in \mathcal{A}_{\gamma, \sigma} \quad(k=1, \ldots, N)\right\}
$$

with norm $\|V\|_{\gamma, \sigma}=\sum_{k=1}^{N}\left\|V_{k}\right\|_{\gamma, \sigma}$.

Let $c=\left(c_{1}, \ldots, c_{N}\right) \in \mathbb{R}^{N}$ and $\alpha$ be a real number satisfying

$$
1<\alpha \leq 2 .
$$

Further, we introduce the space for vector functions $M=\left(M_{1}, \ldots, M_{N}\right)(p)$

$$
\mathcal{M}_{c, \alpha, \sigma}=\left\{M: M(p)=\frac{c}{p}+Z(p) \text { with } Z \in\left(\mathcal{A}_{\alpha, \sigma}\right)^{N}\right\} \quad(\sigma>0)
$$

and the space for scalar functions $B=B(x, p)$

$$
\mathcal{B}_{\gamma, \sigma}=\left\{B: B(x, p) \in \mathcal{A}_{\gamma, \sigma} \text { for } x \in[0,1], B(\cdot, p) \in C[0,1] \text { for } \operatorname{Re} p>\sigma\right\}
$$

with norm $\|B\|_{\gamma, \sigma}=\max _{x \in[0,1]} \sup _{\operatorname{Re} p>\sigma}|p|^{\gamma}|B(x, p)|$. Then we have the following lemma which is a sharpened version of [10: Lemma 2]. 
Lemma 3. Besides (5.5) let the following assumptions be fulfilled:

$\beta, \rho \in C^{2}[0,1]$ and $\beta, \rho>0$ in $[0,1]$

$\mu_{k} \in C^{3}[0,1] \quad(k=1, \ldots, N)$ and $\varphi_{j} \in C^{4}[0,1] \quad(j=1, \ldots, l)$

$\left(\mu_{k} \varphi_{j}^{\prime}\right)^{\prime}(0)=\left(\mu_{k} \varphi_{j}^{\prime}\right)^{\prime}(1)=0 \quad(k=1, \ldots, N ; j=1, \ldots, l)$

$\Lambda_{j} \in \mathcal{B}_{1+\alpha, \sigma_{0}}(j=1, \ldots, l)$ with some $\sigma_{0}>0$.

Then for every $\sigma \geq \sigma_{0}$ and $M \in \mathcal{M}_{c, \alpha, \sigma}$ such that

$$
\eta(M, \sigma):=\left(\frac{1}{\beta_{0}}+C_{2}\right)\|\mu\|\left(\frac{|c|}{\sigma}+\frac{\|Z\|_{\alpha, \sigma}}{\sigma^{\alpha}}\right)<1
$$

where $C_{2}$ is the constant in Lemma $1, \mu=\left(\mu_{1}, \ldots, \mu_{N}\right)$ and

$$
|c|=\sum_{k=1}^{N}\left|c_{k}\right|, \quad \beta_{0}=\min _{x \in[0,1]} \beta(x)>0, \quad\|\mu\|=\max _{1 \leq k \leq N}\left\|\mu_{k}\right\|_{C[0,1]}
$$

equation (5.2) for $j=1, \ldots, l$ has a unique solution $B_{j}=B_{j}[M]$ in $\mathcal{B}_{1+\alpha, \sigma}$. This solution $B_{j}$ satisfies the estimate

$$
\left\|B_{j}\right\|_{1+\alpha, \sigma} \leq \frac{C_{5}}{1-\eta(M, \sigma)}\left(1+\frac{|c|}{\sigma^{2-\alpha}}+\frac{\|Z\|_{\alpha, \sigma}}{\sigma}\right)
$$

with some constant $C_{5}>0$.

Moreover, for every $\sigma \geq \sigma_{0}, M=\frac{c}{p}+Z \in \mathcal{M}_{c, \alpha, \sigma}$ with $\eta(M, \sigma)<1$ and $\widetilde{M}=\frac{c}{p}+\widetilde{Z} \in \mathcal{M}_{c, \alpha, \sigma}$ with $\eta(\widetilde{M}, \sigma)<1$ the difference $B_{j}[M]-B_{j}[\widetilde{M}]$ fulfils the estimate

$$
\begin{aligned}
\| B_{j}[M] & -B_{j}[\widetilde{M}] \|_{1+\alpha, \sigma} \\
\leq & \frac{C_{6}}{1-\eta(\widetilde{M}, \sigma)}\left[\frac{1}{\sigma}+\frac{1}{\sigma^{\alpha}} \frac{1}{1-\eta(M, \sigma)}\left\{1+\frac{|c|}{\sigma^{2-\alpha}}+\frac{\|Z\|_{\alpha, \sigma}}{\sigma}\right\}\right] \\
& \times\|Z-\widetilde{Z}\|_{\alpha, \sigma}
\end{aligned}
$$

with some constant $C_{6}>0$.

Proof. Integral equation (5.2) for $B_{j}=B_{j}[M] \quad(j=1, \ldots, l)$ can be written in the operator form

$$
B_{j}=b_{j}-A_{0} B_{j}
$$

in $\mathcal{B}_{1+\alpha, \sigma}$ where

$$
b_{j}=b_{j}(x, p)=\delta(x, p)\left[\sum_{k=1}^{N} M_{k}(p) \Phi_{j k}(x, p)-\Lambda_{j}(x, p)\right]
$$


and the linear operator $A_{0}: \mathcal{B}_{1+\alpha, \sigma} \rightarrow \mathcal{B}_{1+\alpha, \sigma}$ is defined by

$$
\left(A_{0} B\right)(x, p)=\delta(x, p) \sum_{k=1}^{N} M_{k}(p) \int_{0}^{1} G_{x y}(x, y ; p) \mu_{k}(y) B(y, p) d y
$$

with the factor

$$
\delta(x, p)=\frac{1}{1-\frac{1}{\beta(x)} \sum_{k=1}^{N} M_{k}(p) \mu_{k}(x)} .
$$

Since $|p|>\sigma,|p|^{\alpha}>\sigma^{\alpha}$ for $\operatorname{Re} p>\sigma \geq \sigma_{0}>0$ and $M \in \mathcal{M}_{c, \alpha, \sigma}$, for $\delta$ from (5.16) we have the estimation

$$
|\delta(x, p)| \leq \frac{1}{1-\frac{1}{\beta(x)} \sum_{k=1}^{N}\left|\mu_{k}(x)\right|\left(\frac{\left|c_{k}\right|}{|p|}+\frac{|p|^{\alpha}\left|Z_{k}(p)\right|}{|p|^{\alpha}}\right)} \leq \frac{1}{1-\frac{\|\mu\|}{\beta_{0}} X_{0}(\alpha, \sigma)}
$$

for $\operatorname{Re} p>\sigma$ where

$$
X_{0}(\alpha, \sigma):=\frac{|c|}{\sigma}+\frac{\|Z\|_{\alpha, \sigma}}{\sigma^{\alpha}}<\infty \quad\left(\sigma \geq \sigma_{0}\right)
$$

so that by assumption (5.10) the factor $\delta(x, p)$ in (5.14) and (5.15) is a continuous function holomorphic in $p$ for $\operatorname{Re} p>\sigma$.

To show that $b_{j} \in \mathcal{B}_{1+\alpha, \sigma_{0}}$, we estimate $b_{j}$ from (5.14). By (5.17) and $M \in \mathcal{M}_{c, \alpha, \sigma}$ we have

$$
\begin{aligned}
& |p|^{1+\alpha}\left|b_{j}(x, p)\right| \\
& \leq \frac{1}{1-\frac{\|\mu\|}{\beta_{0}} X_{0}(\alpha, \sigma)}\left[\sum_{k=1}^{N}\left(\left|c_{k}\right|+\frac{|p|^{\alpha}\left|Z_{k}(p)\right|}{|p|^{\alpha-1}}\right)|p|^{\alpha}\left|\Phi_{j k}(x, p)\right|+|p|^{1+\alpha}\left|\Lambda_{j}(x, p)\right|\right]
\end{aligned}
$$

Because of (5.5) the inequalities $|p|^{\alpha-1}>\sigma^{\alpha-1}$ and $|p|^{2-\alpha} \geq \sigma^{2-\alpha}$ for $\operatorname{Re} p>\sigma$ hold. Therefore, we further have

$$
\begin{aligned}
& |p|^{1+\alpha}\left|b_{j}(x, p)\right| \\
& \leq \frac{1}{1-\frac{\|\mu\|}{\beta_{0}} X_{0}(\alpha, \sigma)}\left[\sum_{k=1}^{N}\left(\left|c_{k}\right|+\frac{|p|^{\alpha}\left|Z_{k}(p)\right|}{\sigma^{\alpha-1}}\right) \frac{\left|p^{2} \Phi_{j k}(x, p)\right|}{\sigma^{2-\alpha}}+|p|^{1+\alpha}\left|\Lambda_{j}(x, p)\right|\right] .
\end{aligned}
$$

Applying inequality (4.12) from Lemma 2 to the functions $\Phi_{j k}$ defined by (5.3), which is allowed by assumptions (5.6) - (5.8), we obtain

$$
\left|p^{2} \Phi_{j k}(x, p)\right| \leq C_{3}\left\|\left(\mu_{k} \varphi_{j}^{\prime}\right)^{\prime \prime}\right\|_{C^{1}[0,1]} \quad\left(\operatorname{Re} p \geq \sigma_{0}\right)
$$


and hence

$$
\left\|b_{j}\right\|_{1+\alpha, \sigma} \leq \frac{C}{1-\frac{\|\mu\|}{\beta_{0}} X_{0}(\alpha, \sigma)}\left[\left(|c|+\frac{\|Z\|_{\alpha, \sigma}}{\sigma^{\alpha-1}}\right) \frac{1}{\sigma^{2-\alpha}}+\left\|\Lambda_{j}\right\|_{1+\alpha, \sigma}\right]
$$

follows for $\sigma \geq \sigma_{0}$, with some constant $C>0$. By the holomorphy of $Z$ and $\Lambda_{j}, b_{j}$ is a holomorphic function of $p$ for $\operatorname{Re} p>\sigma$, too. Hence by (5.9) $b_{j} \in \mathcal{B}_{1+\alpha, \sigma}$ for $\sigma \geq \sigma_{0}$ follows.

For estimating the norm of the operator $A_{0}$ given by (5.15) in the space $\mathcal{B}_{1+\alpha, \sigma}$ for $M \in \mathcal{M}_{c, \alpha, \sigma}$ we apply (5.17) and (4.10). We obtain

$$
\begin{aligned}
|p|^{1+\alpha}\left|\left(A_{0} B\right)(x, p)\right| \leq & \frac{1}{1-\frac{\|\mu\|}{\beta_{0}} X_{0}(\alpha, \sigma)}\left[\sum_{k=1}^{N}\left(\left|c_{k}\right|+\frac{|p|^{\alpha}\left|Z_{k}(p)\right|}{\sigma^{\alpha-1}}\right)\right. \\
& \left.\times \frac{1}{\operatorname{Re} p} \int_{0}^{1} \frac{\operatorname{Re} p}{|p|}\left|G_{x y}(x, y ; p)\right|\left|\mu_{k}(y)\right||p|^{1+\alpha}|B(y, p)| d y\right]
\end{aligned}
$$

for $\operatorname{Re} p>\sigma$ and

$$
\begin{aligned}
\left\|A_{0} B\right\|_{1+\alpha, \sigma} & \leq \frac{C_{2}\|\mu\|}{1-\frac{\|\mu\|}{\beta_{0}} X_{0}(\alpha, \sigma)} \frac{1}{\sigma}\left(|c|+\frac{\|Z\|_{\alpha, \sigma}}{\sigma^{\alpha-1}}\right)\|B\|_{1+\alpha, \sigma} \\
& =\frac{C_{2}\|\mu\| X_{0}(\alpha, \sigma)}{1-\frac{\|\mu\|}{\beta_{0}} X_{0}(\alpha, \sigma)}\|B\|_{1+\alpha, \sigma}
\end{aligned}
$$

by (5.18), where $C_{2}$ is the constant in (4.10).

Further, $A_{0} B$ is a holomorphic function of $p$ in $\operatorname{Re} p>\sigma$ for $B \in \mathcal{B}_{1+\alpha, \sigma}$. Thus, $A_{0}$ is a linear bounded operator in $\mathcal{B}_{1+\alpha, \sigma}$, and by (5.20) its norm satisfies the estimate

$$
\left\|A_{0}\right\| \leq \frac{C_{2}\|\mu\| X_{0}(\alpha, \sigma)}{1-\frac{\|\mu\|}{\beta_{0}} X_{0}(\alpha, \sigma)}
$$

so that by (5.18) and assumption (5.10) we have $\left\|A_{0}\right\|<1$. Hence equation (5.13) has a unique solution $B_{j}=B_{j}[M]$ in $\mathcal{B}_{1+\alpha, \sigma}$ for any $M \in \mathcal{M}_{c, \alpha, \sigma}$ satisfying (5.10). Moreover, observing (5.21) with (5.18) and (5.10), we obtain

$$
\left\|B_{j}\right\|_{1+\alpha, \sigma} \leq \frac{\left\|b_{j}\right\|_{1+\alpha, \sigma}}{1-\left\|A_{0}\right\|} \leq \frac{\left\|b_{j}\right\|_{1+\alpha, \sigma}}{1-\eta(M, \sigma)}\left[1-\frac{\|\mu\|}{\beta_{0}} X_{0}(\alpha, \sigma)\right]
$$

and by (5.19) we have the first estimate (5.11). 
It remains to prove assertion (5.12). By equation (5.2),

$$
\begin{aligned}
\left(B_{j}[M]-B_{j}[\widetilde{M}]\right)(x, p) & \\
= & \frac{1}{\beta(x)} \sum_{k=1}^{N} \widetilde{M}_{k}(p) \mu_{k}(x)\left(B_{j}[M]-B_{j}[\widetilde{M}]\right)(x, p) \\
& -\sum_{k=1}^{N} \widetilde{M}_{k}(p) \int_{0}^{1} G_{x y}(x, y ; p) \mu_{k}(y)\left(B_{j}[M]-B_{j}[\widetilde{M}]\right)(y, p) d y \\
& +\sum_{k=1}^{N}\left(M_{k}-\widetilde{M}_{k}\right)(p)\left[\Phi_{j k}(x, p)+\frac{\mu_{k}(x)}{\beta(x)} B_{j}[M](x, p)\right. \\
& \left.-\int_{0}^{1} G_{x y}(x, y ; p) \mu_{k}(y) B_{j}[M](y, p) d y\right] .
\end{aligned}
$$

Estimating this expression analogously as above, in view of inequalities (4.10) of Lemma 1 and (4.12) of Lemma 2 we obtain

$$
\begin{aligned}
& \left\|B_{j}[M]-B_{j}[\widetilde{M}]\right\|_{1+\alpha, \sigma} \\
& \quad \leq \eta(\widetilde{M}, \sigma)\left\|B_{j}[M]-B_{j}[\widetilde{M}]\right\|_{1+\alpha, \sigma}+\bar{C}\left[\frac{1}{\sigma}+\frac{1}{\sigma^{\alpha}}\left\|B_{j}[M]\right\|_{1+\alpha, \sigma}\right]\|Z-\tilde{Z}\|_{\alpha, \sigma}
\end{aligned}
$$

$\left(\sigma \geq \sigma_{0}\right)$ with a constant $\bar{C}>0$. Observing estimation (5.11) for $\left\|B_{j}[M]\right\|_{1+\alpha, \sigma}$ and the assumptions $\eta(M, \sigma)<1$ and $\eta(\widetilde{M}, \sigma)<1$, we get assertion (5.12). Lemma 3 is proved

\section{Existence theorem}

Our main result is the following existence and uniqueness theorem.

Theorem. Let assumptions (5.5) - (5.9) and regularity condition (3.18) for the matrix $\Gamma=\left(\left(\gamma_{n k}\right)\right)$ of the coefficients $\gamma_{n k}$ in (3.13) with relation (3.17) be fulfilled. Moreover, suppose that the vector function $\Psi=\left(\Psi_{1}, \ldots, \Psi_{N}\right)$ of the right-hand sides $\Psi_{n}=\Psi_{j i} \quad(n=1, \ldots, N)$ of (3.13) defined by (3.14) with $n$ given by (3.17) and $N=\sum_{j=1}^{l} N_{j}$ has the representation

$$
\Psi=\frac{d}{p}+Y \in \mathcal{M}_{d, \alpha, \sigma_{0}}
$$

where $d=\left(d_{1}, \ldots, d_{N}\right) \in \mathbb{R}^{N}$ and $Y=\left(Y_{1}, \ldots, Y_{N}\right) \in\left(\mathcal{A}_{\alpha, \sigma_{0}}\right)^{N}$. 
Then there exists a real number $\sigma_{1} \geq \sigma_{0}$ such that the system of equations (3.13) has a unique solution $M=\left(M_{1}, \ldots, M_{N}\right)$ of the form

$$
M=\frac{c}{p}+Z \in \mathcal{M}_{c, \alpha, \sigma_{1}}
$$

where $c=\Gamma^{-1} d$ with $\Gamma^{-1}$ being the inverse of the matrix $\Gamma$.

Proof. The system of equations (3.13) is equivalent to the operator equation

$$
Z=A Z \quad \text { in }\left(\mathcal{A}_{\alpha, \sigma}\right)^{N}
$$

with operator $A=\Gamma^{-1} A_{1}:\left(\mathcal{A}_{\alpha, \sigma}\right)^{N} \rightarrow\left(\mathcal{A}_{\alpha, \sigma}\right)^{N}$ and operator $A_{1}:\left(\mathcal{A}_{\alpha, \sigma}\right)^{N} \rightarrow \mathbb{\square}$ $\left(\mathcal{A}_{\alpha, \sigma}\right)^{N}$ defined by

$$
\begin{aligned}
\left(A_{1} Z\right)_{n}(p)= & Y_{n}(p)+\sum_{k=1}^{N}\left(\frac{c_{k}}{p}+Z_{k}(p)\right) \tilde{\Phi}_{n k}(p) \\
& -\sum_{k=1}^{N}\left(\frac{c_{k}}{p}+Z_{k}(p)\right) \int_{0}^{1} p^{2} G_{y}\left(x_{n}, y ; p\right) \mu_{k}(y) B_{j}\left[\frac{c}{p}+Z\right](y, p) d y
\end{aligned}
$$

for $n=1, \ldots, N$. Here the function $B_{j} \quad(j=1, \ldots, l)$ given by $(5.1)$ is the solution of equation (5.2) and

$$
\tilde{\Phi}_{n k}(p)=\int_{0}^{1} p^{2} G\left(x_{n}, y ; p\right)\left(\mu_{k}(y) \varphi_{j}^{\prime}(y)\right)^{\prime} d y+\frac{1}{\rho\left(x_{n}\right)}\left(\mu_{k}(x) \varphi_{j}^{\prime}(x)\right)^{\prime}\left(x_{n}\right)
$$

for $n, k=1, \ldots, N$, with $x_{n}=x_{i j} \quad\left(i=1, \ldots, N_{j} ; j=1, \ldots, l\right)$ and $n$ related to $i$ and $j$ by (3.17).

For the proof of existence of a unique solution to equation (6.3) we introduce the balls

$$
D_{\alpha, \sigma}(r)=\left\{Z \in\left(\mathcal{A}_{\alpha, \sigma}\right)^{N}:\|Z\|_{\alpha, \sigma} \leq r\right\} \quad(r>0)
$$

for $\sigma \geq 0$ and show that $A$ is a contraction in such a ball. From (6.4) we have

$$
\begin{aligned}
|p|^{\alpha}\left|\left(A_{1} Z\right)_{n}(p)\right| \leq & |p|^{\alpha}\left|Y_{n}(p)\right| \\
& +\sum_{k=1}^{N}\left(\frac{\left|c_{k}\right|}{|p|^{2-\alpha}}+\frac{|p|^{\alpha}\left|Z_{k}(p)\right|}{|p|}\right)\left|p \tilde{\Phi}_{n k}(p)\right| \\
& +\sum_{k=1}^{N}\left(\left|c_{k}\right|+\frac{|p|^{\alpha}\left|Z_{k}(p)\right|}{|p|^{\alpha-1}}\right) \\
& \times \int_{0}^{1}\left|G_{y}\left(x_{n}, y ; p\right)\right|\left|\mu_{k}(y)\right||p|^{1+\alpha}\left|B_{j}\left[\frac{c}{p}+Z\right](y, p)\right| d y .
\end{aligned}
$$


Now due to assumptions (5.6) - (5.8), from assertion (4.13) of Lemma 2 for the functions $\tilde{\Phi}_{n k}(p)$ defined by $(6.5)$ the estimate

$$
\left|p \tilde{\Phi}_{n k}(p)\right| \leq C_{4}\left\|\left(\mu_{k} \varphi_{j}^{\prime}\right)^{\prime \prime}\right\|_{C^{1}[0,1]} \quad(k, n=1, \ldots, N)
$$

for $\operatorname{Re} p>0$ and a constant $C_{4}>0$ holds. By (6.6) and (4.9) of Lemma 1 we further have

$$
\begin{aligned}
|p|^{\alpha}\left|\left(A_{1} Z\right)_{n}(p)\right| \leq & \left\|Y_{n}\right\|_{\alpha, \sigma_{0}}+C_{4} \sum_{k=1}^{N}\left(\frac{\left|c_{k}\right|}{\sigma^{2-\alpha}}+\frac{\left\|Z_{k}\right\|_{\alpha, \sigma}}{\sigma}\right)\left\|\left(\mu_{k} \varphi_{j}^{\prime}\right)^{\prime \prime}\right\|_{C^{1}[0,1]} \\
& +\frac{C_{1}}{\sigma}\|\mu\|\left\|B_{j}\left[\frac{c}{p}+Z\right](y, p)\right\|_{1+\alpha, \sigma} \sum_{k=1}^{N}\left(\left|c_{k}\right|+\frac{\left\|Z_{k}\right\|_{\alpha, \sigma}}{\sigma^{\alpha-1}}\right)
\end{aligned}
$$

$(n=1, \ldots, N)$ for $\operatorname{Re} p>\sigma$ and $\sigma \geq \sigma_{0}$. Moreover, in view of $A=\Gamma^{-1} A_{1}$ and estimate (5.11) we obtain

$$
\begin{aligned}
\|A Z\|_{\alpha, \sigma} \leq & \left|\Gamma^{-1}\right|\left(\|Y\|_{\alpha, \sigma_{0}}+C_{7} \frac{|c|}{\sigma^{2-\alpha}}\right) \\
& +C\left\{\left(\frac{1}{\sigma}+\frac{\|Z\|_{\alpha, \sigma}}{\sigma^{\alpha}}\right) \frac{1}{1-\eta\left(\frac{c}{p}+Z, \sigma\right)}\left[1+\frac{1}{\sigma^{2-\alpha}}+\frac{\|Z\|_{\alpha, \sigma}}{\sigma}\right]\right\}
\end{aligned}
$$

for $\sigma \geq \sigma_{0}$ and $Z$ satisfying $\eta\left(\frac{c}{p}+Z, \sigma\right)=\eta(M, \sigma)<1$ where $\eta(M, \sigma)$ is defined by (5.10), $C>0$ is some constant,

$$
\left|\Gamma^{-1}\right|=\max _{1 \leq n, k \leq N}\left|\left(\Gamma^{-1}\right)_{n k}\right|, \quad C_{7}=C_{4} \max _{1 \leq k \leq N} \sum_{n=1}^{N}\left\|\left(\mu_{k} \varphi_{j}^{\prime}\right)^{\prime \prime}\right\|_{C^{1}[0,1]}
$$

and $j$ is related to $n$ by (3.17) again.

Let now $Z \in D_{\alpha, \sigma}(r)$. Then from (6.7) we have

$$
\begin{aligned}
\|A Z\|_{\alpha, \sigma} \leq & \left|\Gamma^{-1}\right|\left(\|Y\|_{\alpha, \sigma_{0}}+C_{7} \frac{|c|}{\sigma^{2-\alpha}}\right) \\
& +C\left\{\left(\frac{1}{\sigma}+\frac{r}{\sigma^{\alpha}}\right) \frac{1}{1-\eta_{0}(r, \sigma)}\left[1+\frac{1}{\sigma^{2-\alpha}}+\frac{r}{\sigma}\right]\right\}
\end{aligned}
$$

if $\sigma \geq \sigma_{0}$ and $\eta_{0}(r, \sigma):=\left(\frac{1}{\beta_{0}}+C_{2}\right)\|\mu\|\left(\frac{|c|}{\sigma}+\frac{r}{\sigma^{\alpha}}\right)<1$. From estimation (6.8) in view of the assumption $1<\alpha \leq 2$ we see that, for every $r>r_{0}$ where

$$
r_{0}= \begin{cases}\left|\Gamma^{-1}\right|\|Y\|_{\alpha, \sigma_{0}} & \text { for } 1<\alpha<2 \\ \left|\Gamma^{-1}\right|\left(\|Y\|_{\alpha, \sigma_{0}}+C_{7}|c|\right) & \text { for } \alpha=2 \mathrm{~b}\end{cases}
$$


there exists $\sigma_{2}=\sigma_{2}(r)$ such that $\eta_{0}(r, \sigma)<1$ and

$$
\|A Z\|_{\alpha, \sigma} \leq r \quad\left(\sigma \geq \sigma_{2}(r), r>r_{0}\right)
$$

Further, by Lemma 3 we have $B_{j}=B_{j}\left[\frac{c}{p}+Z\right] \in \mathcal{B}_{1+\alpha, \sigma_{2}(r)}$ which implies that the functions $B_{j}=B_{j}(y, p)$ are holomorphic with respect to $p$ on $\operatorname{Re} p>\sigma_{2}(r)$ for $y \in[0,1]$. This together with the holomorphy of $Z \in D_{\alpha, \sigma}(r)$ and the other terms in (6.4) shows the holomorphy of $A Z(p)=\Gamma^{-1} A_{1} Z(p)$ on $\operatorname{Re} p>\sigma_{2}(r)$. Hence by $(6.9)$

$$
A: D_{\alpha, \sigma}(r) \rightarrow D_{\alpha, \sigma}(r) \quad\left(\sigma \geq \sigma_{2}(r), r>r_{0}\right)
$$

follows.

Next we estimate the difference of $A Z^{1}$ and $A Z^{2}$ for $Z^{1}, Z^{2} \in\left(\mathcal{A}_{\alpha, \sigma}\right)^{N}$. From (6.4) we have

$$
\begin{aligned}
\left(A_{1} Z^{1}\right. & \left.-A_{1} Z^{2}\right)_{n}(p) \\
= & \sum_{k=1}^{N}\left(Z_{k}^{1}(p)-Z_{k}^{2}(p)\right) \tilde{\Phi}_{n k}(p) \\
& -\sum_{k=1}^{N}\left(Z_{k}^{1}(p)-Z_{k}^{2}(p)\right) \\
& \times \int_{0}^{1} p^{2} G_{y}\left(x_{n}, y ; p\right) \mu_{k}(y) B_{j}\left[\frac{c}{p}+Z^{1}\right](y, p) d y \\
& -\sum_{k=1}^{N}\left(\frac{c_{k}}{p}+Z_{k}^{2}(p)\right) \\
& \int_{0}^{1} p^{2} G_{y}\left(x_{n}, y ; p\right) \mu_{k}(y)\left(B_{j}\left[\frac{c}{p}+Z^{1}\right]-B_{j}\left[\frac{c}{p}+Z^{2}\right]\right)(y, p) d y .
\end{aligned}
$$

Now let be $Z^{1}, Z^{2} \in D_{\alpha, \sigma}(r)$. Then on account of inequalities (4.9), (4.13) and (5.11) - (5.12), analogously as above we obtain

$$
\begin{aligned}
&\left\|A Z^{1}-A Z^{2}\right\|_{\alpha, \sigma} \\
& \leq \bar{C}\left\{\frac{1}{\sigma}+\frac{1}{\sigma^{\alpha}} \frac{1}{1-\eta_{0}(r, \sigma)}\left[1+\frac{1}{\sigma^{2-\alpha}}+\frac{r}{\sigma}\right]\right. \\
&+\left(\frac{1}{\sigma}+\frac{r}{\sigma^{\alpha}}\right) \frac{1}{1-\eta_{0}(r, \sigma)} \\
&\left.\times\left[\frac{1}{\sigma}+\frac{1}{\sigma^{\alpha}} \frac{1}{1-\eta_{0}(r, \sigma)}\left(1+\frac{1}{\sigma^{2-\alpha}}+\frac{r}{\sigma}\right)\right]\right\}\left\|Z^{1}-Z^{2}\right\|_{\alpha, \sigma}
\end{aligned}
$$


$\left(\sigma \geq \sigma_{0}\right)$ with some constant $\bar{C}>0$. From this estimation, in view of $1<\alpha \leq 2$, again we see that there exists $\sigma_{3}=\sigma_{3}(r)$ such that the operator $A$ is a contraction for $\sigma \geq \sigma_{3}(r)$. This together with (6.10) implies that equation (6.3) has a unique solution in every ball $D_{\alpha, \sigma}(r)$ when $\sigma \geq \sigma_{4}(r)=\max \left\{\sigma_{2}(r), \sigma_{3}(r)\right\}$ and $r>r_{0}$. Therefore, the existence assertion of the theorem is proved for $\sigma_{1}=\sigma_{4}\left(r_{1}\right)$ with some $r_{1}>r_{0}$.

Since any two solutions $Z^{1}, Z^{2}$ of equation (6.3) from $\left(\mathcal{A}_{\alpha, \sigma_{1}}\right)^{N}$ are lying in some common ball $D_{\alpha, \sigma}(r)$ with $\sigma \geq \sigma_{4}(r)$ where $r>\max \left\{\left\|Z^{1}\right\|_{\alpha, \sigma_{1}},\left\|Z^{2}\right\|_{\alpha, \sigma_{1}}, r_{1}\right\}$, the uniqueness of the solution $Z$ in the space $\left(\mathcal{A}_{\alpha, \sigma_{1}}\right)^{N}$ follows from the proven uniqueness in these balls

Corollary. Under the assumptions of Theorem the inverse problem (2.3)(2.7) has the unique solution $m$ of form (2.6) where

$$
m_{k}(t)=c_{k}+\frac{1}{2 \pi i} \int_{\xi-i \infty}^{\xi+i \infty} e^{t p} Z_{k}(p) d p \quad\left(\xi>\sigma_{1}\right)
$$

for $k=1, \ldots, N$ with $c=\left(c_{k}\right)_{k=1}^{N} \in \mathbb{R}^{N}$ and $Z=\left(Z_{k}\right)_{k=1}^{N} \in\left(\mathcal{A}_{\alpha, \sigma_{1}}\right)^{N}$. The functions $m_{k}$ are continuous for $t \geq 0$ and $m_{k}(0)=c_{k}$.

Proof. Since $\alpha>1$ by assumption, this follows from a known inversion formula for the Laplace transformation (cf. [3: Theorem 21.3])

Remark. The inverse problem with additional conditions

$$
\int_{0}^{1} \lambda_{i j}(x) u_{j}(x, t) d t=h_{j i}(t) \quad\left(i=1, \ldots, N_{j} ; j=1, \ldots, l\right)
$$

with prescribed non-negative summable functions $\lambda_{i j}$ instead of (2.7) can be handled in an analogous way as above. If the functions $\lambda_{i j}$ have their supports in a neighbourhood of the points $x_{i j}$ and $\int_{0}^{1} \lambda_{i j}(x) d x=1$, conditions (6.13) above correspond to the measurement of certain mean values of the displacements $u_{j}$ around $x_{i j}$.

\section{References}

[1] Colombo, F. and A. Lorenzi: Identification of time and space dependent relaxation kernels for materials with memory related to cylindrical domains, Part I. J. Math. Anal. Appl. 213 (1997), 32 - 62.

[2] Colombo, F. and A. Lorenzi: Identification of time and space dependent relaxation kernels for materials with memory related to cylindrical domains, Part II. J. Math. Anal. Appl. 213 (1997), 63 - 90. 
[3] Doetsch, G: Einführung in Theorie und Anwendung der Laplace-Transformation Basel: Birkhäuser Verlag 1958.

[4] Grasselli, M: An identification problem for an abstract linear hyperbolic integrodifferential equation with applications. J. Math. Anal. Appl. 171 (1992), 27 -60 .

[5] Grasselli, M: On an inverse problem for a linear hyperbolic integrodifferential equation. Forum Math. 6 (1994), 83 - 110.

[6] Janno, J. and A. Lorenzi: Recovering degenerate kernels in hyperbolic integrodifferential equations. Z. Anal. Anw. (submitted).

[7] Janno, J. and L. von Wolfersdorf: Inverse problems for identification of memory kernels in viscoelasticity. Math. Meth. Appl. Sci. 20 (1997), 291 - 314.

[8] Janno, J. and L. von Wolfersdorf: Identification of weakly singular memory kernels in viscoelasticity. Z. Angew. Math. Mech. 78 (1998), $391-403$.

[9] Janno, J. and L. von Wolfersdorf: Inverse problems for memory kernels by Laplace transform methods. Z. Anal. Anw. 19 (2000), 489 - 510.

[10] Janno, J. and L. von Wolfersdorf: An inverse problem for identification of a time- and space-dependent memory kernel in viscoelasticity. Inverse Problems 17 (2001), $13-24$.

[11] Lorenzi, A. and V. G. Yakhno: An identification problem related to an isotropic nonhomogeneous stratified viscoelastic cylindrical body. J. Inv. Ill-Posed Probl. 5 (1997), $29-53$.

[12] von Wolfersdorf, L.: Inverse problems for memory kernels in heat flow and viscoelasticity. J. Inv. Ill-Posed Probl. 4 (1996), 341 - 354.

Received 13.08.2001 